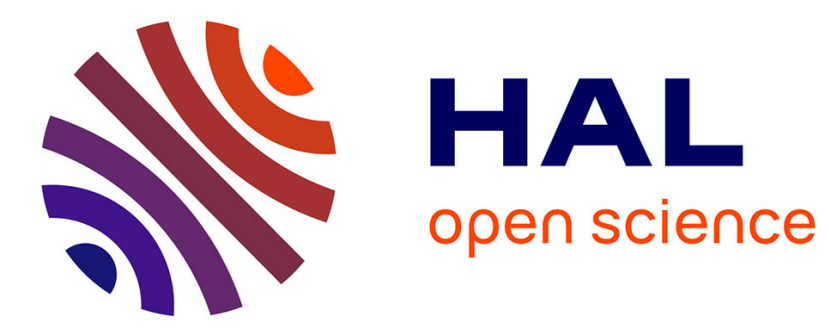

\title{
Non-crossing tree realizations of ordered degree sequences
}

Laurent Mehats, Lutz Strassburger

\section{To cite this version:}

Laurent Mehats, Lutz Strassburger. Non-crossing tree realizations of ordered degree sequences. [Research Report] 2009, pp.15. hal-00649591

\section{HAL Id: hal-00649591 \\ https://hal.inria.fr/hal-00649591}

Submitted on 8 Dec 2011

HAL is a multi-disciplinary open access archive for the deposit and dissemination of scientific research documents, whether they are published or not. The documents may come from teaching and research institutions in France or abroad, or from public or private research centers.
L'archive ouverte pluridisciplinaire HAL, est destinée au dépôt et à la diffusion de documents scientifiques de niveau recherche, publiés ou non, émanant des établissements d'enseignement et de recherche français ou étrangers, des laboratoires publics ou privés. 


\title{
Non-crossing tree realizations of ordered degree sequences
}

\author{
Laurent Méhats \\ LaBRI \& Université de Bordeaux 1 \\ laurent dot mehats at gmail dot com \\ Lutz Straßburger \\ INRIA Saclay \& LIX, École Polytechnique \\ lutz at lix dot polytechnique dot fr
}

December 17, 2009

\begin{abstract}
We investigate the enumeration of non-crossing tree realizations of integer sequences, and we consider a special case in four parameters, that can be seen as a four-dimensional tetrahedron that generalizes Pascal's triangle and the Catalan numbers.
\end{abstract}

\section{Introduction}

Enumeration of non-crossing tree realizations of integer compositions A non-crossing tree $t$ is a labeled tree on a sequence of vertices $\left\langle v_{0}, v_{1}, \ldots, v_{n}\right\rangle$ drawn in counterclockwise order on a circle, and whose edges are straight line segments that do not cross. For any index $0 \leq i \leq n$, let $d_{i}$ stand for the number of edges incident with $v_{i}$ (that is the degree of $v_{i}$ ). Then as any other tree on $n+1$ vertices, $t$ satisfies $\sum_{i=0}^{n} d_{i}=2 n$. Thus, the sequence $\left\langle d_{0}, d_{1}, \ldots, d_{n}\right\rangle$ defines a composition of $2 n$ into $n+1$ positive summands (two sequences of integers that differ only in the order of their elements define distinct compositions of the same integer). Stated otherwise, $t$ is a non-crossing tree realization of the composition $\left\langle d_{0}, d_{1}, \ldots, d_{n}\right\rangle$.

For any composition $c=\left\langle d_{0}, d_{1}, \ldots, d_{n}\right\rangle$ of $2 n$ into $n+1$ positive summands, let $n c t(c)$ stand for the number of non-crossing tree realizations of $c$, that is the number of non-crossing trees on $n+1$ vertices $\left\langle v_{0}, v_{1}, \ldots, v_{n}\right\rangle$ such that vertex $v_{i}$ has degree $d_{i}$ for any index $0 \leq i \leq n$ (there always exists at least one, see Proposition 2.3). We aim at computing nct. Note that here the input is more specific than the degree partition, as for example in [5].

From proof nets to non-crossing trees Our interest for these non-crossing tree realizations comes from linguistics and proof theory. The starting point for this work was the following linguistic problem: How many different readings can an ambiguous sentence at most have? Particularly, which sentence of a given length has the most different readings? When using categorial grammars based on the Lambek calculus [9] or related systems, a parse tree is a formal proof in a deductive system. Thus, our questions become: How many different formal proofs can a formula have? Particularly, which formula of a given length has the most different formal proofs? In category theoretical terms these questions come down to the cardinality of the Hom-sets in a free non-commutative star-autonomous category [2]. The corresponding logic is a variant of non-commutative intuitionistic linear logic $[16,8]$ for which formal proofs can be represented as planar proof nets. It would go too far beyond the scope of this paper to go into the details of this correspondence. However, to give the reader an idea, we have shown in Figure 1 the transformation of a parse tree into a proof net. The first step transforms the parse tree into a formal proof according to Lambek's work [9]. In the second step, this proof is embedded into a one-sided multiple conclusion system using the 


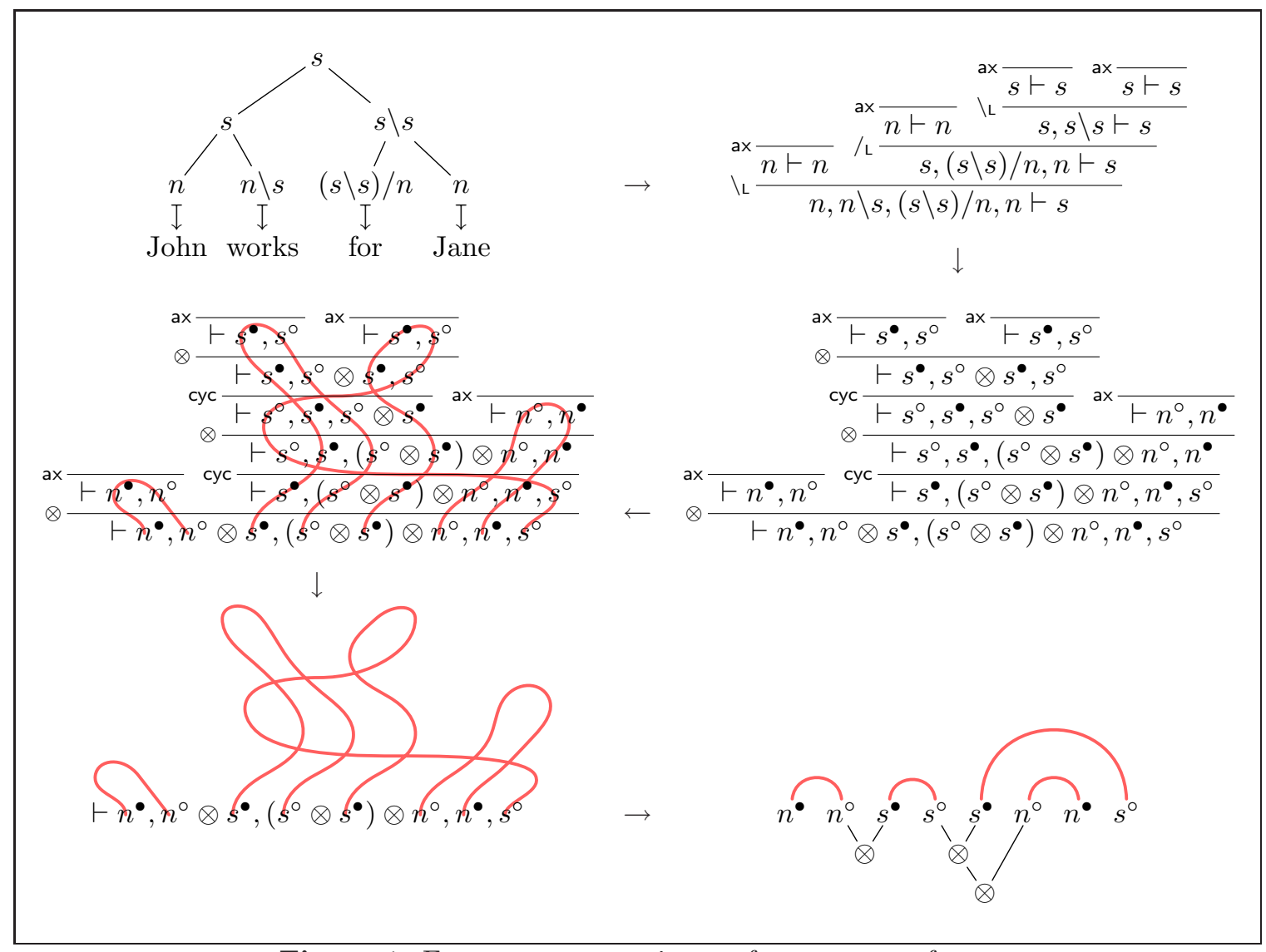

Figure 1: From parse trees via proof trees to proof nets

binary connectives 8 and $\otimes[16]$. In order not to lose the information on positive and negative positions in the formulas we use polarities (see, e.g., [8] for details). The final three steps show how this one-sided sequent proof is translated into a proof net by simply drawing the flow graph on the atoms appearing in the proof (for more details, see [8, 3, 14]). It is a well-known fact of linear logic that such a graph $G$ does indeed correspond to a sequent proof if and only if every switching (that is, every graph obtained from $G$ by removing for each 8 -node one of the two edges that it to its children) is a connected and acyclic graph [4]. If $G$ does not contain any 8-nodes, as in our example, then $G$ itself has to be connected and acyclic. Furthermore, we have that $G$ is planar if and only if the sequent proof does not contain the exchange rule, as it is the case for the Lambek calculus [16].

Thus, our questions become: How many different planar proof nets can at most be defined over a given sequent? Particularly, over which sequent of a given length can the most different planar proof nets be defined?

In that respect, we can ignore the names of the atoms, and only 8 -free sequents are of interest: on the one hand, occurrences of 8 lying above an occurrence of $\otimes$ can moved down by the transformations

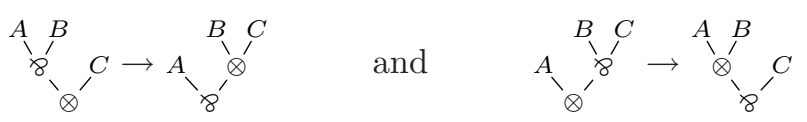

which preserve correctness without affecting linkings (see $[6,7]$ ); on the other hand, root occurrences of $>$ are irrelevant and can be removed. Hence, for every sequent $\Gamma$ there is a 8 -free sequent $\Gamma^{\prime}$, such that for $\Gamma^{\prime}$ exist at least as many different planar proof nets as for $\Gamma$.

Finally, up to the associativity of $\otimes$, planar 8 -free proof nets are in bijection with non-crossing trees as shown in Figure 2.

We were not able to find a closed formula for $n c t\left\langle d_{0}, d_{1}, \ldots, d_{n}\right\rangle$ depending only on the input composition $\left\langle d_{0}, d_{1}, \ldots, d_{n}\right\rangle$. However experiments show that at least up to $n=25$, nct is 


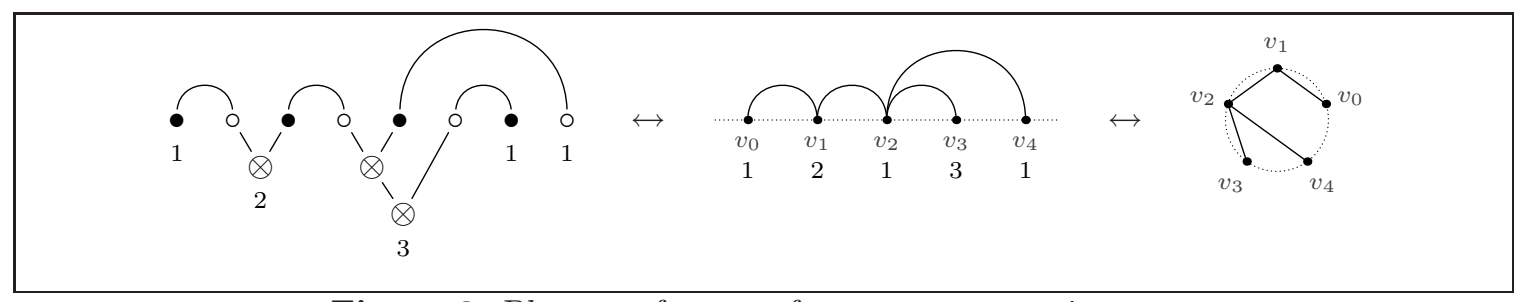

Figure 2: Planar 8 -free proof nets as non-crossing trees

maximized for compositions are of the shape

$$
1, \underbrace{2,2, \ldots, 2}_{p \text { summands }}, \underbrace{1,3,1,3, \ldots, 1,3}_{2 q \text { summands }}, 1, \underbrace{2,2, \ldots, 2}_{r \text { summands }}, \underbrace{1,3,1,3, \ldots, 1,3}_{2 s \text { summands }}
$$

which we write as $12^{p}(13)^{q} 12^{r}(13)^{s}$ and are the first step of our study (compositions maximizing nct for higher values of $n$ may be of a different shape or involve summands higher then 3 ). The input is now reduced to four parameters $p, q, r$ and $s$ such that $n+1=1+p+2 q+1+r+2 s$. We write $n c t\left\langle 12^{p}(13)^{q} 12^{r}(13)^{s}\right\rangle$ as $N_{p, q, r, s}$, and we are interested in computing $N_{p, q, r, s}$.

A four-dimensional Generalization of Pascal's and Catalan's triangles Recall that Pascal's triangle $P_{p, r}=\left(\begin{array}{c}p+r \\ p, r\end{array}\right)$ can be generated recursively by:

$$
P_{p, r}= \begin{cases}1 & \text { if } p=0 \text { or } r=0 \\ P_{p, r-1}+P_{p-1, r} & \text { if } p>0 \text { and } r>0\end{cases}
$$

The first few values are shown below (see [13, A007318]):

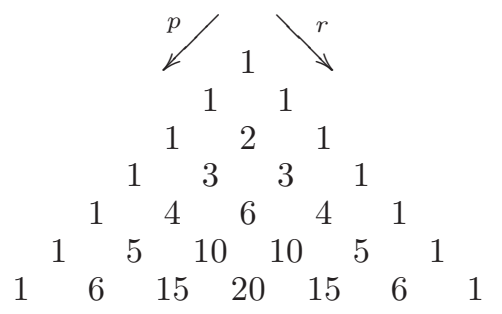

The Catalan numbers $C_{q}=\frac{1}{q+1}\left(\begin{array}{c}2 q \\ q, q\end{array}\right)$ are generated recursively by $C_{0}=1$ and $C_{q+1}=\sum_{j=0}^{q} C_{j}$. $C_{q-j}$. A combination of Pascal's triangle and the Catalan numbers is known as Catalan's triangle $Q_{p, q}=\frac{p+1}{p+q+1}\left(\begin{array}{c}p+2 q \\ p+q, q\end{array}\right)$ which can be generated recursively by:

$$
Q_{p, q}= \begin{cases}1 & \text { if } q=0 \\ C_{q} & \text { if } p=0 \\ Q_{p+1, q-1}+Q_{p-1, q} & \text { if } p, q>0\end{cases}
$$

The first few values are shown below (see [13, A009766]):

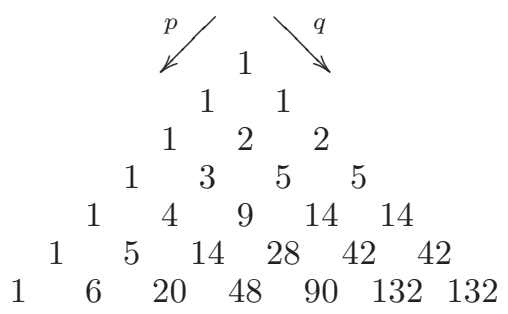


It is also possible to generalize the recursive formula of the Catalan numbers into a triangle $R_{q, s}$ generated by (assuming that $C_{-1}=0$ ):

$$
R_{q, s}= \begin{cases}1 & \text { if } q=s=0 \\ \sum_{j=0}^{q} \sum_{l=0}^{s} C_{j+l-1} \cdot R_{q-j, s-l} & \text { if } q+s>0\end{cases}
$$

The first few values are shown below:

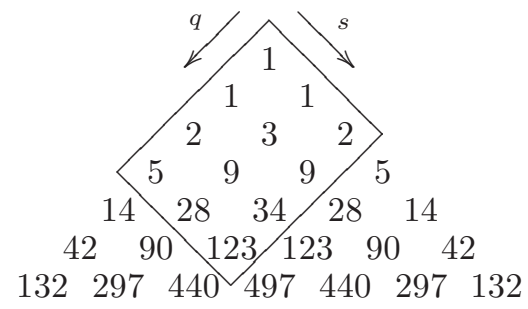

As an example,

$$
123=C_{0} \cdot(28+34)+C_{1} \cdot(5+9+9)+C_{2} \cdot(2+3+2)+C_{3} \cdot(1+1)+C_{4} \cdot 1 .
$$

We shall establish that $N_{p, q, r, s}$ is a four-dimensional "tetrahedron" that generalizes the three triangles $P, Q$ and $R$ above, insofar as:

$$
\begin{aligned}
& N_{p, 0, r, 0}=N_{r, 0, p, 0}=P_{p, r}, \\
& N_{p, q, 0,0}=N_{0,0, p, q}=Q_{p, q}, \\
& N_{0, q, 0, s}=N_{0, s, 0, q}=R_{q, s} .
\end{aligned}
$$

Outline The organization of this paper is as follows: First, in Section 2, we study the general case of enumerating non-crossing tree realizations of integer compositions. Then, in Sections 37, we concentrate on the four-parameter case. In particular, we will prove identities (8)-(10) in Sections 4 and 5. Finally, we will provide the generating function for $N_{p, q, r, s}$ in Section 7.

\section{General case}

Any labeled tree on a sequence of vertices can be drawn in such a way that its vertices lie in counterclockwise order on a circle and its edges are straight line segments lying inside that circle. In that case, of course, some of its edges may cross each other. Let us call such a labeled tree a crossing tree. The order of summands in a composition does not matter regarding the number of its labeled tree realizations (there are six for any composition of $2 \cdot 4$ into $4+1$ summands in the multiset $\{1,1,2,2,2\})$. But it does as soon as we distinguish between non-crossing and crossing realizations. As an example, there are one non-crossing and five crossing realizations of $\langle 1,1,2,2,2\rangle$, while there are three non-crossing and three crossing realizations of $\langle 1,2,1,2,2\rangle$ (these are shown on Figure 3).

Remark 2.1. A proof of Cayley's formula (see e.g., [1]), which asserts that the number of labeled trees on $n+1$ vertices is $(n+1)^{n-1}$ (see [13, A000272]) relies on:

$$
\sum_{\left\langle d_{0}, d_{1}, \ldots, d_{n}\right\rangle}\left(\begin{array}{c}
n-1 \\
d_{0}-1, d_{1}-1, \ldots, d_{n}-1
\end{array}\right)=(n+1)^{n-1}
$$

where the sum ranges over the $\left(\begin{array}{c}2 n-1 \\ n, n-1\end{array}\right)$ compositions of $2 n$ into $n+1$ positive summands. Noy established in [10, Corollary 1.2] that the number of non-crossing trees on $n+1$ vertices is $\frac{1}{2 n+1}\left(\begin{array}{c}3 n \\ 2 n, n\end{array}\right)$ (see $[13, \mathrm{~A} 001764])$.) Recall that $n c t\left\langle d_{0}, d_{1}, \ldots, d_{n}\right\rangle$ stands for the number of non-crossing tree realizations of the composition $n c t\left\langle d_{0}, d_{1}, \ldots, d_{n}\right\rangle$. Then

$$
\sum_{\left\langle d_{0}, d_{1}, \ldots, d_{n}\right\rangle} n c t\left\langle d_{0}, d_{1}, \ldots, d_{n}\right\rangle=\frac{1}{2 n+1}\left(\begin{array}{c}
3 n \\
2 n, n
\end{array}\right)
$$



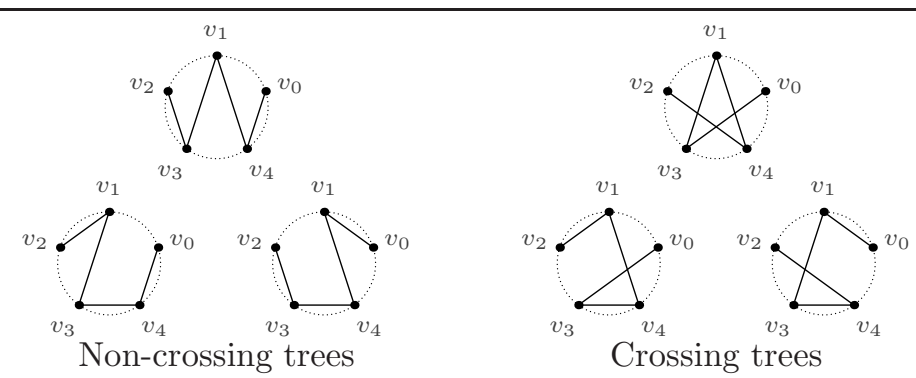

Figure 3: The six labeled tree realizations of $\langle 1,2,1,2,2\rangle$

where the sum ranges over the $\left(\begin{array}{c}2 n-1 \\ n, n-1\end{array}\right)$ compositions of $2 n$ into $n+1$ positive summands.

The image under rotation of a non-crossing tree $t$ on vertices $\left\langle v_{0}, v_{1}, \ldots, v_{n}\right\rangle$ is a non-crossing tree on vertices $\left\langle v_{\sigma(0)}, v_{\sigma(1)}, \ldots, v_{\sigma(n)}\right\rangle$ for some cyclic permutation $\sigma$ in the symmetric group $S_{n+1}$. Moreover, $t$ realizes a composition $\left\langle d_{0}, d_{1}, \ldots, d_{n}\right\rangle$ iff its image under rotation realizes the composition $\left\langle d_{\sigma(0)}, d_{\sigma(1)}, \ldots, d_{\sigma(n)}\right\rangle$. Thus, for any composition $\left\langle d_{0}, d_{1}, \ldots, d_{n}\right\rangle$ and any cyclic permutation $\sigma \in S_{n+1}$,

$$
n c t\left\langle d_{0}, d_{1}, \ldots, d_{n}\right\rangle=n c t\left\langle d_{\sigma(0)}, d_{\sigma(1)}, \ldots, d_{\sigma(n)}\right\rangle
$$

We shall refer to this property as stability under rotation. ${ }^{1}$

The same way, the mirror image of a non-crossing tree $t$ on vertices $\left\langle v_{0}, v_{1}, \ldots, v_{n}\right\rangle$ is a noncrossing tree on vertices $\left\langle v_{n}, v_{n-1}, \ldots, v_{0}\right\rangle$, and $t$ realizes a composition $\left\langle d_{0}, d_{1}, \ldots, d_{n}\right\rangle$ iff its mirror image realizes the composition $\left\langle d_{n}, d_{n-1}, \ldots, d_{0}\right\rangle$. Thus, for any composition $\left\langle d_{0}, d_{1}, \ldots, d_{n}\right\rangle$,

$$
n c t\left\langle d_{0}, d_{1}, \ldots, d_{n}\right\rangle=n c t\left\langle d_{n}, d_{n-1}, \ldots, d_{0}\right\rangle
$$

We shall refer to this property as stability under mirror image. ${ }^{2}$

We will now establish that for any positive integer $n$ and any composition $c$ of $2 n$ into $n+1$ positive summands, there exists a non-crossing tree realization of $c$ (Proposition 2.3).

Lemma 2.2. For any positive integer $n$ and any sequence $\left\langle 1, d_{1}, \ldots, d_{n}, d_{n+1}\right\rangle$ of $n+2$ positive integers such that $1+\sum_{i=1}^{n+1} d_{i}<2(n+1)$, there is an index $1 \leq k<n+1$ such that $1+\sum_{i=1}^{k} d_{i}=2 k$.

Proof. For any index $1 \leq l \leq n+1$, let $S_{l}$ stand for $1+\sum_{i=1}^{l} d_{i}$. We prove the following implication by induction on $l$ : if there is no index $1 \leq k<l$ such that $S_{k}=2 k$, then $S_{l} \geq 2 l$. Since by hypothesis $S_{n+1}<2(n+1)$, there must exist an index $1 \leq k<n+1$ such that $S_{k}=2 k$.

Base. Since $d_{1} \geq 1, S_{1}=1+d_{1} \geq 2 \cdot 1$ and the stated implication holds trivially.

Induction. Assume that the stated implication holds for $l(\mathrm{IH})$, and that there exists no index $1 \leq k<l+1$ such that $S_{k}=2 k$. We reformulate the latter hypothesis as: (i) there exists no index $1 \leq k<l$ such that $S_{k}=2 k$, and (ii) $S_{l} \neq 2 l$. By (IH) we get from (i), that $S_{l} \geq 2 l$, and from (ii), that $S_{l}>2 l$, i.e., $S_{l} \geq 2 l+1$. Since $d_{l+1} \geq 1, S_{l+1}=S_{l}+d_{l+1} \geq 2(l+1)$.

Proposition 2.3. For any positive integer $n$ and any composition $c$ of $2 n$ into $n+1$ positive summands, there exists a non-crossing tree realization of $c$.

Proof. We proceed by induction on $n$.

Base. The unique composition $\langle 1,1\rangle$ of $2 \cdot 1$ into $1+1$ positive summands is realized by the unique (trivially non-crossing) tree on $1+1$ vertices.

Induction. Assume that the stated property holds for any positive integer up to $n$ (IH), and let $\left\langle d_{0}, d_{1}, \ldots, d_{n+1}\right\rangle$ be a composition of $2(n+1)$ into $n+2$ positive summands. Since $n$ is a positive integer, $2(n+1)>n+2$ and there must exist at least one summand $d_{k}>1$. By stability under

\footnotetext{
${ }^{1}$ In that respect, we may focus on necklace-compositions, i.e., compositions that are lexicographically minimal under rotation [12].

${ }^{2}$ In that respect, we may focus on bracelet-compositions, i.e., necklace-compositions that are lexicographically minimal under mirror image [11].
} 


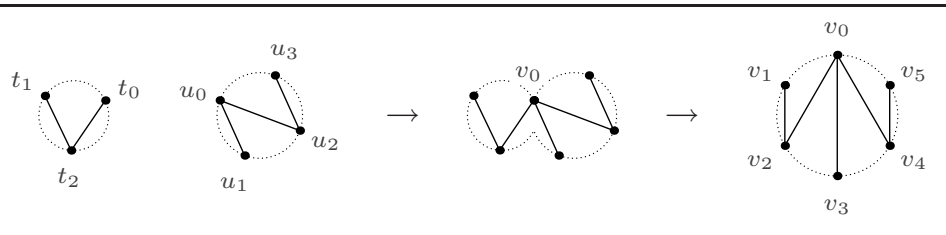

Figure 4: Merging two non-crossing trees into a single one

rotation, we can assume without loss of generality that $d_{0}$ is such a summand, i.e. that $d_{0}>1$. Then $1+\sum_{i=1}^{n+1} d_{i}<2(n+1)$ and by Lemma 2.2 , there exists an index $1 \leq k<n+1$ such that $1+\sum_{i=1}^{k} d_{i}=2 k$. By difference, $\left(d_{0}-1\right)+\sum_{i=k+1}^{n+1} d_{i}=2(n-k+1)$. Then by (IH):

- there exists a non-crossing tree on vertices $\left\langle t_{0}, t_{1}, \ldots, t_{k}\right\rangle$ realizing the composition $\left\langle 1, d_{1}, \ldots, d_{k}\right\rangle$ of $2 k$ into $k+1$ positive summands,

- there exists a non-crossing tree on vertices $\left\langle u_{0}, u_{1}, \ldots, u_{n-k+1}\right\rangle$ realizing the composition $\left\langle d_{0}-1, d_{k+1}, \ldots, d_{n+1}\right\rangle$ of $2(n-k+1)$ into $n-k+2$ positive summands.

Let $T$ and $U$ stand for the respective edge sets of these non-crossing trees (where edges are defined as couples of vertices). We "merge" $t_{0}$ and $u_{0}$ into a single vertex $v_{0}$ to get a tree on vertices $\left\langle v_{0}, v_{1}, \ldots, v_{n+1}\right\rangle$ which edge set is defined as

$$
\begin{aligned}
& \left\{\left\{v_{i}, v_{j}\right\} \mid\left\{t_{i}, t_{j}\right\} \in T\right\} \\
\cup & \left\{\left\{v_{0}, v_{j+k}\right\} \mid\left\{u_{0}, u_{j}\right\} \in U\right\} \\
\cup & \left\{\left\{v_{i+k}, v_{j+k}\right\} \mid\left\{u_{i}, u_{j}\right\} \in U, i>0, j>0\right\}
\end{aligned}
$$

(see Figure 4). This tree is non-crossing and it realizes the composition $\left\langle d_{0}, d_{1}, \ldots, d_{n}, d_{n+1}\right\rangle$ of $2(n+1)$ into $n+2$ positive summands.

The previous proof suggests a recursive definition of nct:

- The unique composition $\langle 1,1\rangle$ of $2 \cdot 1$ into $1+1$ positive summands is realized by the unique non-crossing tree on $1+1$ vertices. Thus,

$$
n c t\langle 1,1\rangle=1 .
$$

- Let $n$ be strictly greater than 1 and $\left\langle d_{0}, d_{1}, \ldots, d_{n}\right\rangle$ be a composition of $2 n$ into $n+1$ positive summands. Let $k$ be the smallest index such that $d_{k}>1$ (there exists at least one). By stability under rotation,

$$
n c t\left\langle 1, \ldots, 1, d_{k}, \ldots, d_{n}\right\rangle=\operatorname{nct}\left\langle d_{k}, \ldots, d_{n}, 1, \ldots, 1\right\rangle .
$$

Thus we can assume that $d_{0}>1$. In that case,

$$
n c t\left\langle d_{0}, d_{1}, \ldots, d_{n}\right\rangle=\sum_{k}\left(n c t\left\langle 1, d_{1}, \ldots, d_{k}\right\rangle \cdot \operatorname{nct}\left\langle d_{0}-1, d_{k+1}, \ldots, d_{n}\right\rangle\right)
$$

where the sum ranges over the set of indices $1 \leq k<n$ such that $1+\sum_{i=1}^{k} d_{i}=2 k$ (there exists at least one).

Remark 2.4. Applying the recursive formula in a row to $d_{0}, d_{0}-1, \ldots, 1$, we get

$$
n c t\left\langle d_{0}, d_{1}, \ldots, d_{n}\right\rangle=\sum_{\left\langle k_{0}, \ldots, k_{d_{0}}\right\rangle} \prod_{j=1}^{d_{0}} n c t\left\langle 1, d_{k_{j-1}+1}, \ldots, d_{k_{j}}\right\rangle
$$

where the sum ranges over the set of sequences $\left\langle k_{0}, \ldots, k_{d_{0}}\right\rangle$ of $d_{0}+1$ indices such that $0=k_{0}<$ $\cdots<k_{d_{0}}=n$ and such that for all $0<j \leq d_{0}$,

$$
1+\sum_{i=k_{j-1}+1}^{k_{j}} d_{i}=2\left(k_{j}-k_{j-1}\right) .
$$




\section{The four parameters case}

We focus now on the special case where compositions are of the shape

$$
1, \underbrace{2,2, \ldots, 2}_{p}, \underbrace{1,3,1,3, \ldots, 1,3}_{2 q}, 1, \underbrace{2,2, \ldots, 2}_{r}, \underbrace{1,3,1,3, \ldots, 1,3}_{2 s}
$$

which we write as $12^{p}(13)^{q} 12^{r}(13)^{s}$. Recall that $N_{p, q, r, s}$ stands for $\operatorname{nct}\left\langle 12^{p}(13)^{q} 12^{r}(13)^{s}\right\rangle$.

Lemma 3.1. For any $p, q, r$ and $s, N_{p, q, r, s}=N_{r, q, p, s}=N_{p, s, r, q}$.

Proof. This follows from stability under rotation and mirror image. We give the formal calculations here in full, because we use similar arguments later on without showing them explicit.

$$
\begin{aligned}
N_{p, q, r, s} & =n c t\left\langle 12^{p}(13)^{q} 12^{r}(13)^{s}\right\rangle & & \text { by definition of } N \\
& =n c t\left\langle 2^{p}(13)^{q} 12^{r}(13)^{s} 1\right\rangle & & \text { by stability under rotation } \\
& =n c t\left\langle 2^{p} 1(31)^{q} 2^{r} 1(31)^{s}\right\rangle & & \text { by reparenthesizing } \\
& =n c t\left\langle(13)^{s} 12^{r}(13)^{q} 12^{p}\right\rangle & & \text { by stability under mirror image } \\
& =n c t\left\langle 12^{r}(13)^{q} 12^{p}(13)^{s}\right\rangle & & \text { by stability under rotation } \\
& =N_{r, q, p, s} & & \text { by definition of } N .
\end{aligned}
$$

The same way, $N_{p, q, r, s}=N_{p, s, r, q}$.

According to Lemma 3.1, so as to get a recursive definition of $N_{p, q, r, s}$, we need to consider only $N_{0,0,0,0}$ on the one hand, $N_{p+1, q, r, s}$ and $N_{p, q, r, s+1}$ on the other hand.

Proposition 3.2. For any $p, q, r$ and $s$,

$$
\begin{aligned}
N_{0,0,0,0} & =1 \\
N_{p+1, q, r, s} & =N_{0,0,0,0} \cdot N_{p, q, r, s}+\sum_{j=1}^{q}\left(N_{0, j, 0,0} \cdot N_{p, q-j, r, s}\right) \\
& +\sum_{k=1}^{r}\left(N_{0, q, k, 0} \cdot N_{p, 0, r-k, s}\right)+\sum_{l=1}^{s}\left(N_{0, q, r, l} \cdot N_{p, 0,0, s-l}\right) \\
N_{p, q, r, s+1} & =N_{0,0,0,0} \cdot N_{1+p, q, r, s}+\sum_{i=1}^{p}\left(N_{i, 0,0,0} \cdot N_{1+p-i, q, r, s}\right)+\sum_{j=1}^{q}\left(N_{p, j, 0,0} \cdot N_{1, q-j, r, s}\right)
\end{aligned}
$$

Proof. We have $N_{0,0,0,0}=\operatorname{nct}\left\langle 12^{0}(13)^{0} 12^{0}(13)^{0}\right\rangle=1$ by definition of $N$ and (17). Next we have $N_{p+1, q, r, s}=\operatorname{nct}\left\langle 12^{p} 2(13)^{q} 12^{r}(13)^{s}\right\rangle=\operatorname{nct}\left\langle 21(31)^{q} 2^{r}(13)^{s} 12^{p}\right\rangle$ by definition of $N$, reparenthesizing and stability under rotation. Applying (19) we get

$$
\begin{aligned}
N_{p+1, q, r, s} & =\operatorname{nct}\langle 11\rangle \cdot \operatorname{nct}\left\langle 1(31)^{q} 2^{r}(13)^{s} 12^{p}\right\rangle \\
& +\sum_{j=1}^{q}\left(\operatorname{nct}\left\langle 11(31)^{j}\right\rangle \cdot \operatorname{nct}\left\langle 1(31)^{q-j} 2^{r}(13)^{s} 12^{p}\right\rangle\right) \\
& +\sum_{k=1}^{r}\left(\operatorname{nct}\left\langle 11(31)^{q} 2^{k}\right\rangle \cdot \operatorname{nct}\left\langle 12^{r-k}(13)^{s} 12^{p}\right\rangle\right) \\
& +\sum_{l=1}^{s}\left(\operatorname{nct}\left\langle 11(31)^{q} 2^{r}(13)^{l}\right\rangle \cdot \operatorname{nct}\left\langle 1(13)^{s-l} 12^{p}\right\rangle\right) .
\end{aligned}
$$

Notice that there is no other way to "split" $\left\langle 21(31)^{q} 2^{r}(13)^{s} 12^{p}\right\rangle$ into two compositions such that the first one is of the form $\left\langle 1, d_{1}, \ldots, d_{k}\right\rangle$ and satisfies $1+\sum_{i=1}^{k} d_{i}=2 k$. Then we get $(24)$ by reparenthesizing and stability under rotation. A similar argument applies to the proof of (25).

Corollary 3.3. For any $p$ and $s$,

$$
\begin{aligned}
N_{p, 0,0,0} & =1 \\
N_{0,0,0, s} & =C_{s} \\
N_{0,0, r, s+1} & =N_{1,0, r, s}
\end{aligned}
$$

where $C_{s}$ stands for the $s$-th Catalan number.

Proof. Both (26) and (27) are easily proved by induction, and (28) follows from (25) and (26). 


\section{Pascal's \& Catalan's triangles}

In this section we are going to establish identities (8) and (9) mentioned in the introduction. First, recall Pascal's second identity and its consequence: for any $a, b$ and $c$,

$$
\sum_{k=0}^{b}\left(\begin{array}{c}
a+k \\
a, k
\end{array}\right)=\left(\begin{array}{c}
a+1+b \\
a+1, b
\end{array}\right) \text { and } \sum_{k=c+1}^{b}\left(\begin{array}{c}
a+k \\
a, k
\end{array}\right)=\left(\begin{array}{c}
a+1+b \\
a+1, b
\end{array}\right)-\left(\begin{array}{c}
a+1+c \\
a+1, c
\end{array}\right)
$$

Proposition 4.1. For all $p, r$ and $s$,

$$
\begin{aligned}
N_{p, 0, r, 0} & =\left(\begin{array}{c}
p+r \\
p, r
\end{array}\right)=P_{p, r} \\
N_{p, 0,0, s} & =\left(\begin{array}{c}
p+2 s \\
p+s, s
\end{array}\right)-\frac{s}{p+s+1}\left(\begin{array}{c}
p+2 s \\
p+s, s
\end{array}\right) \\
& =\frac{p+1}{p+s+1}\left(\begin{array}{c}
p+2 s \\
p+s, s
\end{array}\right)=Q_{p, s} \\
N_{p, 0, r, s} & =\left(\begin{array}{c}
p+r+2 s \\
p+s, r+s
\end{array}\right)-\frac{s}{p+r+s+1}\left(\begin{array}{c}
p+r+2 s \\
p+r+s, s
\end{array}\right)
\end{aligned}
$$

Proof. First we prove (30) by induction on $p$, then we prove (33) by induction on $s$. From this (31) and (32) follow as a special case with $r=0$. By Lemma 3.1 and (26) we have for any $r$ that $N_{0,0, r, 0}=N_{r, 0,0,0}=1$. Now assume that $N_{p, 0, r, 0}=\left(\begin{array}{c}p+r \\ p, r\end{array}\right)$ for any $r$ (IH1). Then

$$
\begin{aligned}
N_{p+1,0, r, 0} & =\sum_{k=0}^{r} N_{0,0, k, 0} \cdot N_{p, 0, r-k, 0}=\sum_{k=0}^{r} N_{p, 0, r-k, 0} & & \text { by }(24) \\
& =\sum_{k=0}^{r}\left(\begin{array}{c}
p+r-k \\
p, r-k
\end{array}\right) & & \text { by (IH1) } \\
& =\left(\begin{array}{c}
p+1+r \\
p+1, r
\end{array}\right) & & \text { by reindexing and (29). }
\end{aligned}
$$

Then (30) holds, and trivially $N_{p, 0, r, 0}=\left(\begin{array}{c}p+r+2 \cdot 0 \\ p+0, r+0\end{array}\right)-\frac{0}{p+r+0+1}\left(\begin{array}{c}p+r+2 \cdot 0 \\ p+r+0,0\end{array}\right)$. Now assume that (33) holds for any $p$ and $r$, i.e. that $N_{p, 0, r, s}=\left(\begin{array}{c}p+r+2 s \\ p+s, r+s\end{array}\right)-\frac{s}{p+r+s+1}\left(\begin{array}{c}p+r+2 s \\ p+r+s, s\end{array}\right)=\left(\begin{array}{c}p+r+2 s \\ p+s, r+s\end{array}\right)-\left(\begin{array}{c}p+r+2 s \\ p+r+s+1, s-1\end{array}\right)$ (IH2). Then

$$
\begin{aligned}
N_{p, 0, r, s+1} & =\sum_{i=1}^{p+1} N_{i, 0, r, s} & & \text { by }(25),(26) \text { and reindexing } \\
& =\sum_{i=1}^{p+1}\left(\begin{array}{c}
i+r+2 s \\
i+s, r+s
\end{array}\right)-\sum_{i=1}^{p+1}\left(\begin{array}{c}
i+r+2 s \\
i+r+s+1, s-1
\end{array}\right) & & \text { by (IH2) } \\
& \left.=\left[\begin{array}{c}
p+r+2 s+2 \\
p+s+1, r+s+1
\end{array}\right)-\left(\begin{array}{c}
r+2 s+1 \\
s, r+s+1
\end{array}\right)\right]-\left[\left(\begin{array}{c}
p+r+2 s+2 \\
p+r+s+2, s
\end{array}\right)-\left(\begin{array}{c}
r+2 s+1 \\
r+s+1, s
\end{array}\right)\right] & & \text { by reindexing and (29) }
\end{aligned}
$$

and the last term is equal to $\left(\begin{array}{c}p+r+2(s+1) \\ p+(s+1), r+(s+1)\end{array}\right)-\frac{s+1}{p+r+(s+1)+1}\left(\begin{array}{c}p+r+2(s+1) \\ p+r+(s+1), s+1\end{array}\right)$.

\section{A triangular Catalan recurrence}

In this section we establish the identity (10) from the introduction. We need the following lemma:

Lemma 5.1. For any $t$ and $u$,

$$
\sum_{i=0}^{t} \sum_{j=0}^{u} C_{i} \cdot C_{j} \cdot C_{(t-i)+(u-j)}=C_{t+u+1} .
$$

Proof. By induction on $u$ (the base case is the usual recurrence for Catalan numbers). 
Proposition 5.2 (A recurrence for $N_{0, q, 0, s}$ ). For any $q$ and $s$,

$$
\begin{aligned}
N_{1, q, 0, s} & =\sum_{j=0}^{q} \sum_{l=0}^{s} C_{j+l} \cdot N_{0, q-j, 0, s-l} \\
N_{2, q, 0, s} & =\sum_{j=0}^{q} \sum_{l=0}^{s} C_{j} \cdot C_{l} \cdot N_{1, q-j, 0, s-l} \\
& =\sum_{j=0}^{q} \sum_{l=0}^{s} C_{j+l+1} \cdot N_{0, q-j, 0, s-l} \\
N_{0, q+1,0, s+1} & =N_{1, q+1,0, s}+N_{1, q, 0, s+1}-N_{2, q, 0, s}
\end{aligned}
$$

Hence $N_{0, q, 0, s}=R_{q, s}$.

Proof. Note that (39) follows from (23), (35), (37) and (38). Hence, it suffices to prove (35)-(38).

First step. Starting from (24) and (25) we prove that for any $q$ and $s$,

$$
N_{1, q, 0, s+1}=\sum_{j=0}^{q} C_{j} \cdot N_{0, q-j, 0, s+1}+\sum_{j=0}^{q} \sum_{l=0}^{s} C_{j} \cdot C_{l} \cdot N_{1, q-j, 0, s-l} .
$$

Second step. Then we prove by induction on $s$ that for any $q$ and $s$, both (35) and the following identity holds:

$$
\sum_{j=0}^{q} \sum_{l=0}^{s} C_{j} \cdot C_{l} \cdot N_{1, q-j, 0, s-l}=\sum_{j=0}^{q} \sum_{l=0}^{s} C_{j+l+1} \cdot N_{0, q-j, 0, s-l} .
$$

The base case for (35) follows from (24) and the base case for (41) from (25). Then the inductive case for (35) follows from (40) and the inductive case for (41) from (34).

Third step. By (25), reindexing and (24) we get that for any $q$ and $s$,

$$
\begin{aligned}
\sum_{j=0}^{q} C_{j} \cdot N_{0, q-j, 0, s+1} & =\sum_{j=0}^{q} N_{1, j, 0,0} \cdot N_{1, q-j, 0, s} \\
& =\sum_{j=0}^{q} C_{j+1} \cdot N_{1, q-j, 0, s}
\end{aligned}
$$

where (43) follows from (42) and $N_{1, q, 0,0}=\sum_{j=0}^{q} C_{j} \cdot C_{q-j}=C_{q+1}$.

Fourth step. We prove (36) and (37). By (25), $N_{1, q, 0, s+1}=N_{2, q, 0, s}+\sum_{j=0}^{q} N_{1, j, 0,0} \cdot N_{1, q-j, 0, s}$.

$$
\begin{array}{rlrl}
N_{2, q, 0, s} & =N_{1, q, 0, s+1}-\sum_{j=0}^{q} N_{1, j, 0,0} \cdot N_{1, q-j, 0, s} & \\
& =N_{1, q, 0, s+1}-\sum_{j=0}^{q} C_{j} \cdot N_{0, q-j, 0, s+1} & & \text { by (42) } \\
& =N_{1, q, 0, s+1}-\left(N_{1, q, 0, s+1}-\sum_{j=0}^{q} \sum_{l=0}^{s} C_{j} \cdot C_{l} \cdot N_{1, q-j, 0, s-l}\right) & & \text { by (40) } \\
& =\sum_{j=0}^{q} \sum_{l=0}^{s} C_{j} \cdot C_{l} \cdot N_{1, q-j, 0, s-l}=\sum_{j=0}^{q} \sum_{l=0}^{s} C_{j+l+1} \cdot N_{0, q-j, 0, s-l} & & \text { by (41) }
\end{array}
$$

Fifth step. Finally, we prove (38).

$$
\begin{aligned}
N_{0, q+1,0, s+1} & =N_{1, q+1,0, s}+\sum_{j=0}^{q} C_{j+1} \cdot N_{1, q-j, 0, s} & & \text { by }(25) \text { and reindexing } \\
& =N_{1, q+1,0, s}+\sum_{j=0}^{q} C_{j} \cdot N_{0, q-j, 0, s+1} & & \text { by }(43) \\
& =N_{1, q+1,0, s}+\left(N_{1, q, 0, s+1}-\sum_{j=0}^{q} \sum_{l=0}^{s} C_{j} \cdot C_{l} \cdot N_{1, q-j, 0, s-l}\right) & & \text { by (40) } \\
& =N_{1, q+1,0, s}+\left(N_{1, q, 0, s+1}-\sum_{j=0}^{q} \sum_{l=0}^{s} C_{j+l+1} \cdot N_{0, q-j, 0, s-l}\right) & & \text { by (41) } \\
& =N_{1, q+1,0, s}+\left(N_{1, q, 0, s+1}-N_{2, q, 0, s}\right) & & \text { by (37) }
\end{aligned}
$$




\section{Triangles and Tetrahedra}

The value of $N_{p, q, r, s}$ depends on four parameters $p, q, r, s$. If we fix two of them, we can obtain triangles. For example, for $q=s=0$ we get Pascal's triangle (3), and for $r=s=0$ we get the Catalan triangle (5) (which should more precisely be called Pascal-Catalan triangle). If we let $p=r=0$, then we get the triangle (7), which could also be called Catalan triangle.

If we fix only one parameter, we obtain a tetrahedron. For example, let $s=0$, and let us define $T_{p, q, r}=N_{p, q, r, 0}$. Then we get from (33) and Lemma 3.1:

$$
T_{p, q, r}=\left(\begin{array}{c}
p+r+2 q \\
p+q, r+q
\end{array}\right)-\frac{q}{p+r+q+1}\left(\begin{array}{c}
p+r+2 q \\
p+r+q, q
\end{array}\right)
$$

This defines a tetrahedron where one side is Pascal's triangle and the other two sides are the Catalan triangle. Thus we can call it the Pascal-Catalan tetrahedron. We have the following recursive identities:

Proposition 6.1. For all $p$, $q$, and $r$, we have

$$
\begin{aligned}
T_{p+1, q+1, r+1} & =T_{p+1, q+1, r}+T_{p, q+1, r+1}+T_{(p+1)+(r+1), q, 0} \\
T_{p+1, q, r+1} & =T_{p, q+1, r}+T_{(p+1)+(r+1), q, 0} \\
T_{p+1, q+1, r} & =T_{p+2, q, r}+T_{p, q+1, r}
\end{aligned}
$$

Proof. Easy calculation using (44).

For $r=0$, we get the tetrahedron $V_{p, q, s}=N_{p, q, 0, s}$, which we can call the Catalan tetrahedron, because two of its sides are the Catalan triangle (5) and the third side is the new Catalan triangle (7). Unfortunately, we could not find a closed formula for $V_{p, q, s}$. However, in Section 7 we will give the generating function. We also have the following:

Proposition 6.2. For all $p, q$, and $s$, we have:

$$
\sum_{i=2}^{p+2} V_{i, q, s}=\sum_{j=0}^{q} C_{j} \cdot V_{p, q+1-j, s}
$$

Proof. By (24) we get $V_{p+1, q, s+1}=\sum_{j=0}^{q} V_{0, j, 0} \cdot V_{p, q-j, s+1}+\sum_{l=1}^{s+1} V_{0, q, l} \cdot V_{p, 0,(s+1)-l}$, where

$$
\begin{aligned}
& \sum_{j=0}^{q} V_{0, j, 0} \cdot V_{p, q-j, s+1} \\
& =\sum_{j=0}^{q} V_{0, j, 0} \cdot\left[\sum_{i=0}^{p-1} V_{1+p-i, q-j, s}+\sum_{j^{\prime}=0}^{q-j} V_{p, j^{\prime}, 0} \cdot V_{1, q-j-j^{\prime}, s}\right] \text { by }(25) \\
& =\sum_{j=0}^{q} V_{0, j, 0} \cdot \sum_{i=0}^{p-1} V_{1+p-i, q-j, s}+\sum_{j=0}^{q} V_{p+1, j, 0} \cdot V_{1, q-j, s} \quad \text { by reindexing and (24) } \\
& \sum_{l=1}^{s+1} V_{0, q, l} \cdot V_{p, 0,(s+1)-l} \\
& =\sum_{l=0}^{s}\left[\sum_{j=0}^{q} V_{0, j, 0} \cdot V_{1, q-j, s}\right] \cdot V_{p, 0, s-l} \\
& =\sum_{j=0}^{q} V_{0, j, 0} \cdot\left[V_{p,(q-j)+1, s}-\sum_{i=0}^{p-1} V_{1+p-i, q-j, s}\right]
\end{aligned}
$$

Hence, we get

$$
V_{p+1, q, s+1}=\sum_{j=0}^{q} V_{p+1, j, 0} \cdot V_{1, q-j, s}+\sum_{j=0}^{q} V_{0, j, 0} \cdot V_{p,(q-j)+1, s}
$$

We also have $V_{p+1, q, s+1}=\sum_{i=2}^{p+2} V_{i, q, s}+\sum_{j=0}^{q} V_{p+1, j, 0} \cdot V_{1, q-j, s}$ by (25) and reindexing. Together with (49), this yields (48).

Observe that identities (45)-(47) establish close relationships among the triangles with $s=0$ (or $q=0$ ), i.e., the triangles that live inside the tetrahedron $T_{p, q, r}$. For example, from (46) we can get $N_{p+1, q, 7,0}=N_{p, q+1,6,0}+N_{p+8, q, 0,0}$. 
Below, we exhibit some identities between triangles where $q \neq 0$ and $s \neq 0$.

$$
\begin{aligned}
N_{1, q, 1, s} & =N_{0, q, 0, s+1}+N_{0, q+1,0, s} \\
N_{1, q, 2, s} & =N_{0, q+1,0, s+1} \\
N_{1, q+1,2, s+1} & =N_{2, q, 2, s}+N_{1, q, 1, s+2}+N_{1, q+2,1, s} \\
N_{1, q+1,0, s+1} & =N_{0, q+1,0, s+1}+N_{3, q, 1, s} \\
N_{1, q+1,1, s+1} & =N_{2, q+1,0, s+1}+N_{2, q, 0, s} \\
N_{p, q, 0,1} & =\sum_{i=2}^{p+2} N_{i, q, 0,0}
\end{aligned}
$$

They can all be proved by using Lemma 3.1, (24) and (25) by easy but tedious calculations.

Now we can derive another recurrence for the triangle in (7), i.e., different from the one given in (6):

Proposition 6.3. For all $q$ and $s$, we have

$$
R_{q, s+2}=\sum_{j=0}^{q} C_{j+1} \cdot\left(R_{q-j, s+1}+R_{q+1-j, s}\right)
$$

Proof. We have

$$
\begin{aligned}
N_{0, q, 0, s+2} & =\sum_{j=0}^{q} N_{0, j, 0,0} \cdot N_{1, q-j, 0, s+1} & & \text { by }(25) \\
& =\sum_{j=0}^{q} \sum_{l=0}^{q-j} N_{0, j, 0,0} \cdot N_{0, l, 0,0} \cdot N_{1, q-j-l, 1, s} & & \text { by Lemma } 3.1 \text { and }(25) \\
& =\sum_{j=0}^{q} \sum_{l=0}^{q-j} C_{j} \cdot C_{l} \cdot\left(N_{0, q-j-l, 0, s+1}+N_{0, q-j-l+1,0, s}\right) & & \text { by }(27) \text { and }(50) \\
& =\sum_{j=0}^{q} C_{j+1} \cdot\left(N_{0, q-j, 0, s+1}+N_{0, q-j+1,0, s}\right) & &
\end{aligned}
$$

To see an example for (56) consider again the triangle (7):

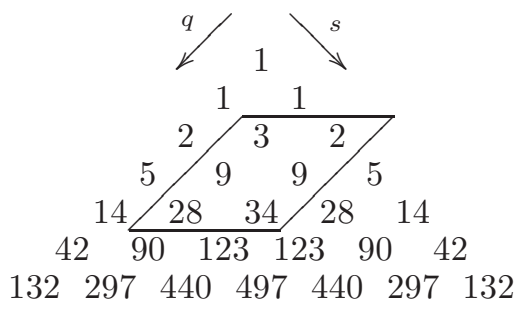

We have $123=C_{1} \cdot(28+34)+C_{2} \cdot(9+9)+C_{3} \cdot(3+2)$.

Remark 6.4. In the next section we will make use of the following identity:

$$
N_{2, q, 0, s}=\sum_{j=0}^{q} N_{0, j, 0,0} \cdot N_{0, q+1-j, 0, s}=\sum_{l=0}^{s} N_{0,0,0, l} \cdot N_{0, q, 0, s+1-l}
$$

It is a special case of (48), but it can also be shown directly: By (36), we have

$$
N_{2, q, 0, s}=\sum_{j=0}^{q} \sum_{l=0}^{s} C_{j} \cdot C_{l} \cdot N_{1, q-j, 0, s-l}
$$

Then (58) follows immediately by (25) and (27).

In the remainder of this section we derive a closed formula for the tetrahedron $s=1$. We need the following observation:

Lemma 6.5. For any $p, r, s$ and $t$,

$$
N_{p, 0, r, s+t}+N_{p+r+t, 0, t, s}=N_{p+t, 0, r+t, s}+N_{p+r, 0,0, s+t}
$$


Proof. Easy calculation, using (33).

For any $t$ and $n$, let $U_{t}(n)$ be defined as

$$
U_{t}(n)=\frac{1}{t+2 n}\left(\begin{array}{c}
t+2 n \\
t+n, n
\end{array}\right) .
$$

Notice that for any $t$,

$$
t U_{t}(0)=\frac{t}{t}\left(\begin{array}{c}
t \\
t, 0
\end{array}\right)=1
$$

The following two identities are called Rothe's identities (see [15, identities 14-15, p. 329]).

$$
\sum_{k=0}^{n} U_{t}(k) \cdot U_{u}(n-k)=\frac{t+u}{t u} U_{t+u}(n) \quad \text { and } \quad \sum_{k=0}^{n} k U_{t}(k) \cdot U_{u}(n-k)=\frac{n}{u} U_{t+u}(n) .
$$

As a consequence, we get

$$
\sum_{k=0}^{n}(t+k) U_{t}(k) \cdot u U_{u}(n-k)=(t+u+n) U_{t+u}(n) .
$$

Lemma 6.6. For any $p, r$ and $s$,

$$
\begin{aligned}
& N_{p, 0, r, s}=(p+s+1) U_{p-r+1}(r+s)-s U_{p+r+1}(s) \\
& N_{p, 0,0, s}=(p+1) U_{p+1}(s)
\end{aligned}
$$

Proof. The identity (64) follows immediately from (33) and (60). The identity (65) is a special case of (64).

Lemma 6.7. For any $p, r, s$ and $t$,

$$
\sum_{l=0}^{s} N_{p, 0, r, l} \cdot N_{t, 0,0, s-l}=N_{p+t+1,0, r, s}-\sum_{l=0}^{r-1}(p-r+1+l) U_{p-r+1}(l) \cdot(t+1) U_{t+1}(r+s-l)
$$

Proof. From (64) and (65) we get that $\sum_{l=0}^{s} N_{p, 0, r, l} \cdot N_{t, 0,0, s-l}$ is equal to

$$
\sum_{l=0}^{s}(p+l+1) U_{p-r+1}(r+l) \cdot(t+1) U_{t+1}(s-l)-\sum_{l=0}^{s} l U_{p+r+1}(l) \cdot(t+1) U_{t+1}(s-l)
$$

where, using (63) and (62) respectively,

$$
\begin{aligned}
& \sum_{l=0}^{s}(p+l+1) U_{p-r+1}(r+l) \cdot(t+1) U_{t+1}(s-l) \\
& =((p+t+1)+s+1) U_{(p+t+1)-r+1}(r+s) \\
& -\sum_{l=0}^{r-1}((p-r+1)+l) U_{p-r+1}(l) \cdot(t+1) U_{t+1}((r+s)-l) \\
& \sum_{l=0}^{s} l U_{p+r+1}(l) \cdot(t+1) U_{t+1}(s-l)=s U_{(p+t+1+r+1}(s)
\end{aligned}
$$

But by $(64),((p+t+1)+s+1) U_{(p+t+1)-r+1}(r+s)-s U_{(p+t+1)+r+1}(s)=N_{p+t+1,0, r, s}$.

Now we can give a closed formula for the $N_{p, q, r, 1}$ tetrahedron.

Proposition 6.8. For all $p, q$ and $r$,

$$
\begin{aligned}
N_{p, q, r, 1} & =T_{p, q+1, r}+T_{p+r+1, q+1,0}-T_{0, q+1, r}-T_{p, q+1,0} \\
& =T_{p+1, q, r+1}+T_{p+r, q, 1}-T_{1, q, r}-T_{p, q, 1}
\end{aligned}
$$

Proof. By (25) we have

$$
N_{p, q, r, 1}=\left(\sum_{i=0}^{p} N_{i, 0,0,0} \cdot N_{1+p-i, q, r, 0}\right)-N_{p, 0,0,0} \cdot N_{1, q, r, 0}+\sum_{j=0}^{q} N_{p, j, 0,0} \cdot N_{1, q-j, r, 0}
$$

where $\sum_{i=0}^{p} N_{i, 0,0,0} \cdot N_{1+p-i, q, r, 0}=N_{p, q+1, r, 0}$ by Lemma 3.1 and (25), and $N_{p, 0,0,0} \cdot N_{1, q, r, 0}=N_{1, q, r, 0}$ by (26), and

$$
\begin{aligned}
\sum_{j=0}^{q} N_{p, j, 0,0} \cdot N_{1, q-j, r, 0} & =\sum_{j=0}^{q}\left(N_{p, 0,0, q-j} \cdot N_{r, 0,1, j}\right) & & \text { by Lemma } 3.1 \text { an } \\
& =N_{p+r+1,0,1, q}-r U_{r}(0) \cdot(p+1) U_{p+1}(q+1) & & \text { by }(66) \\
& =N_{p+r+1,0,1, q}-1 \cdot N_{p, 0,0, q+1} & & \text { by }(61) \text { and }(65)
\end{aligned}
$$

Now both (67) and (68) follow from Lemma 3.1, (28), and (59). 


\section{Generating functions}

We can use the identities (24) and (58) for calculating the generating function for $N_{p, q, r, s}$. Recall that we use the following abbreviations:

$$
\begin{array}{rlrl}
P_{p, r} & =N_{p, 0, r, 0} & C_{q} & =N_{0, q, 0,0} \\
Q_{p, q} & =N_{p, q, 0,0} \\
R_{q, s} & =N_{0, q, 0, s} & T_{p, q, r} & =N_{p, q, r, 0} \\
V_{p, q, s} & =N_{p, q, 0, s}
\end{array}
$$

Theorem 7.1. We have

$$
C(y)=\sum_{q} C_{q} y^{q}=\frac{1-\sqrt{1-4 y}}{2 y}
$$

(ii) $\quad P(x, z)=\sum_{p, r} P_{p, r} x^{p} z^{r}=\frac{1}{1-x-z}$

(iii) $\quad Q(x, y)=\sum_{p, q} Q_{p, q} x^{p} y^{q}=\frac{C(y)}{1-x \cdot C(y)}$

(iv) $\quad R(y, w)=\sum_{q, s} R_{q, s} y^{q} w^{s}=\frac{C(y) \cdot C(w) \cdot(w-y)}{w \cdot C(y)-y \cdot C(w)}$

(v) $\quad T(x, y, z)=\sum_{p, q, r} T_{p, q, r} x^{p} y^{q} z^{r}=\frac{(1-x-z-x \cdot z \cdot C(y)) \cdot C(y)}{(1-x \cdot C(y)) \cdot(1-z \cdot C(y)) \cdot(1-x-z)}$

$$
=P(x, z) \cdot(1+y \cdot Q(x, y) \cdot Q(z, y))
$$

(vi) $\quad V(x, z, w)=\sum_{p, q, s} V_{p, q, s} x^{p} y^{q} w^{s}=\frac{C(y) \cdot C(w) \cdot(w-y-x \cdot(w \cdot C(y)-y \cdot C(w)))}{(w \cdot C(y)-y \cdot C(w)) \cdot(1-x \cdot C(y)) \cdot(1-x \cdot C(w))}$

$$
=Q(x, y) \cdot Q(x, w) \cdot\left(\frac{R(y, w)}{C(y) \cdot C(w)}-x\right)
$$

(vii) $\quad N(x, y, z, w)=\sum_{p, q, r, s} N_{p, q, r, s} x^{p} y^{q} z^{r} w^{s}$

$$
\begin{aligned}
& =\frac{C(y) C(w)\left((1-x-z)(w-y)+(w C(y)-y C(w))\left((1-x-z)(x z(C(y)+C(w))-x-z)+x^{2} z^{2} C(y) C(w)\right)\right)}{(1-x C(y))(1-x C(w))(1-z C(y))(1-z C(w))(1-x-z)(w C(y)-y C(w))} \\
& =Q(x, y) Q(x, y) Q(x, y) Q(x, y)\left(\frac{R(y, w)}{C(y)^{2} \cdot C(w)^{2}}+x^{2} z^{2} P(x, z)-\frac{x}{C(w) Q(z, y)}-\frac{z}{C(y) Q(x, w)}\right)
\end{aligned}
$$

Proof. The formulas in (i) and (ii) are well-known. For the others, the calculation follows below.

(iii) By letting $r=s=0$ in (24) and using (69) we get

$$
Q_{p+1, q}=\sum_{i=0}^{q} Q_{0, i} \cdot Q_{p, q-i}
$$

If we plug (70) into the general formula for $Q(x, y)$, we get

$$
\begin{aligned}
Q(x, y) & =\sum_{q} Q_{0, q} y^{q}+x \sum_{p, q}\left(\sum_{i=0}^{q} C_{i} \cdot Q_{p, q-i}\right) x^{p} y^{q} \\
& =C(y)+x \cdot C(y) \cdot Q(x, y)
\end{aligned}
$$

From this, (iii) follows immediately.

(iv) We let

$$
R^{\prime}(y, w)=\sum_{q, s} R_{q+1, s} y^{q} w^{s} \quad \text { and } \quad R^{\prime \prime}(y, w)=\sum_{q, s} R_{q, s+1} y^{q} w^{s}
$$

Then, we get immediately from (58) and (69)

$$
C(y) \cdot R^{\prime}(y, w)=C(w) \cdot R^{\prime \prime}(y, w)
$$


Furthermore, we have

$$
R(y, w)=\sum_{s} R_{0, s} w^{s}+y \sum_{q, s} R_{q+1, s} y^{q} w^{s}=C(w)+x \cdot R^{\prime}(y, w)
$$

Hence, we get

$$
R^{\prime}(y, w)=\frac{R(y, w)-C(w)}{y} \quad \text { and } \quad R^{\prime \prime}(y, w)=\frac{R(y, w)-C(y)}{w}
$$

Plugging (72) into (71) and solving for $R(y, w)$ yields (iv).

(v) We let $s=0$ in (24) and use (69):

$$
T_{p+1, q, r}=\sum_{i=0}^{q} T_{0, i, 0} \cdot T_{p, q-i, r}+\sum_{j=0}^{r} T_{0, q, j} \cdot T_{p, 0, r-j}-T_{0, q, 0} \cdot T_{p, 0, r}
$$

If we plug (73) into the general formula for $T(x, y, z)$, we get

$$
\begin{aligned}
T(x, y, z) & =\sum_{q, r} T_{0, q, r} y^{q} z^{r}+x \sum_{p, q, r}\left(\sum_{i=0}^{q} C_{i} \cdot T_{p, q-i, r}+\sum_{j=0}^{r} Q_{j, q} \cdot P_{p, r-j}-C_{q} \cdot P_{p, r}\right) x^{p} y^{q} z^{r} \\
& =Q(z, y)+x \cdot C(y) \cdot T(x, y, z)+x \cdot Q(z, y) \cdot P(x, z)-x \cdot C(y) \cdot P(x, z)
\end{aligned}
$$

By plugging in (ii) and (iii), and solving for $T(x, y, z)$, we get the first identity in (v), which can be transformed into

$$
T(x, y, z)=\frac{1}{1-x-z}\left(1-\frac{1-C(y)}{(1-x \cdot C(y))(1-z \cdot C(y))}\right)
$$

By applying the identity $y C(y)^{2}=1-C(y)$ and (ii) and (iii), we get the second identity in $(\mathrm{v})$.

(vi) We let $r=0$ in (24) and use (69):

$$
V_{p+1, q, s}=\sum_{i=0}^{q} V_{0, i, 0} \cdot V_{p, q-i, s}+\sum_{k=0}^{s} V_{0, q, k} \cdot V_{p, 0, s-k}-V_{0, q, 0} \cdot V_{p, 0, s}
$$

If we plug (74) into the general formula for $V(x, y, w)$, we get

$$
\begin{aligned}
& V(x, y, w) \\
& =\sum_{q, s} V_{0, q, s} y^{q} w^{s}+x \sum_{p, q, s}\left(\sum_{i=0}^{q} C_{i} \cdot V_{p, q-i, s}+\sum_{k=0}^{s} R_{q, k} \cdot Q_{p, s-k}-C_{q} \cdot Q_{p, s}\right) x^{p} y^{q} w^{s} \\
& =R(y, w)+x \cdot C(y) \cdot V(x, y, w)+x \cdot R(y, w) \cdot Q(x, w)-x \cdot C(y) \cdot Q(x, w)
\end{aligned}
$$

We get the first line in (v) by plugging (iii) and (iv) into (75), and solving for $V(x, y, z)$. The equality to the second line is obtained by a straightforward calculation.

(vii) From (24) and (69) we get

$$
\begin{aligned}
N_{p+1, q, r, s} & =\sum_{j=0}^{q} N_{0, j, 0,0} \cdot N_{p, q-j, r, s}+\sum_{k=0}^{r} N_{0, q, k, 0} \cdot N_{p, 0, r-k, s}-N_{0, q, 0,0} \cdot N_{p, 0, r, s} \\
& +\sum_{l=0}^{s} N_{0, q, r, l} \cdot N_{p, 0,0, s-l}-N_{0, q, r, 0} \cdot N_{p, 0,0, s} \\
& =\sum_{j=0}^{q} C_{j} \cdot N_{p, q-j, r, s}+\sum_{k=0}^{r} Q_{k, q} \cdot T_{p, r-k, s}-C_{q} \cdot T_{p, r, s} \\
& +\sum_{l=0}^{s} V_{r, q, l} \cdot Q_{p, s-l}-Q_{r, q} \cdot Q_{p, s}
\end{aligned}
$$

If we plug this into the general formula

$N(x, y, z, w)=\sum_{p, q, r, s} N_{p, q, r, s} x^{p} y^{q} z^{r} w^{s}=\sum_{q, r, s} N_{0, q, r, s} y^{q} z^{r} w^{s}+x \sum_{p, q, r, s} N_{p+1, q, r, s} x^{p} y^{q} z^{r} w^{s}$ we get

$$
\begin{aligned}
N(x, y, z, w)=V(x, y, w) & +x \cdot C(y) \cdot N(x, y, z, w) \\
& +x \cdot Q(z, y) \cdot T(x, w, z)-x \cdot C(y) \cdot T(x, w, z) \\
& +x \cdot V(x, y, w) \cdot Q(x, w)-x \cdot Q(z, y) \cdot Q(x, w)
\end{aligned}
$$

If we solve (76) for $N(x, y, z, w)$, and plug in (iii), (v), and (vi), we get the first term in (vii). The equality to the second term is obtained via a straightforward calculation and (ii), (iii), and (iv). 


\section{Acknowledgments}

We thank Mireille Bousquet-Mélou and Gilles Schaeffer for helpful discussions.

\section{References}

[1] Martin Aigner and Günter M. Ziegler, Proofs from THE BOOK, 3rd ed., Springer, 2003.

[2] Michael Barr, Non-symmetric *automomous categories, Theoretical Computer Science 139 (1995), $115-130$.

[3] Richard Blute, Linear logic, coherence and dinaturality, Theoretical Computer Science 115 (1993), $3-41$.

[4] Vincent Danos and Laurent Regnier, The structure of multiplicatives, Annals of Mathematical Logic 28 (1989), 181-203.

[5] Philippe Flajolet and Marc Noy, Analytic combinatorics of non-crossing configurations, Discrete Mathematics 204 (1999), no. 1-3, 203-229, doi:10.1016/S0012-365X(98)00372-0.

[6] Alessio Guglielmi, A system of interaction and structure, ACM Transactions on Computational Logic 8 (2007), no. 1.

[7] Alessio Guglielmi and Lutz Straßburger, Non-commutativity and MELL in the calculus of structures, Computer Science Logic, CSL 2001 (Laurent Fribourg, ed.), LNCS, vol. 2142, Springer-Verlag, 2001, pp. 54-68.

[8] François Lamarche and Christian Retoré, Proof nets for the Lambek-calculus - an overview, Proceedings of the Third Roma Workshop "Proofs and Linguistic Categories" (V. Michele Abrusci and Claudia Casadio, eds.), CLUEB, Bologna, 1996, pp. 241-262.

[9] Joachim Lambek, The mathematics of sentence structure, American Mathematical Monthly 65 (1958), 154-169.

[10] Marc Noy, Enumeration of noncrossing trees on a circle, Discrete Mathematics 180 (1998), no. 1-3, 301-313, doi:10.1016/S0012-365X(97)00121-0.

[11] Joe Sawada, Generating bracelets in constant amortized time, SIAM J. Comput. 31 (2001), no. 1, 259-268.

[12] _ A fast algorithm to generate necklaces with fixed content, Theoretical Computer Science 301 (2003), no. 1-3, 477-489.

[13] Neil J. A. Sloane, The On-Line Encyclopedia of Integer Sequences, 2008, http://www.research.att.com/ njas/sequences/.

[14] Lutz Straßburger, Proof nets and the identity of proofs, Research Report 6013, INRIA, 102006 , Lecture notes for ESSLLI'06.

[15] Volker Strehl, Identities of Rothe-Abel-Schläfli-Hurwitz-type, Discrete Mathematics 99 (1992), no. 1-3, 321-340, doi:10.1016/0012-365X(92)90379-T.

[16] David N. Yetter, Quantales and (noncommutative) linear logic, Journal of Symbolic Logic 55 (1990), no. $1,41-64$. 